\title{
Fractional Factorial Treatment of Ring Spun Yarn Quality
}

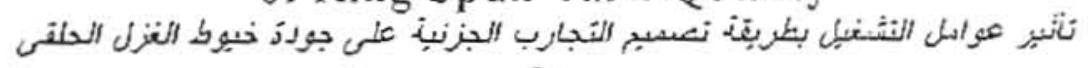
By

Dr. Fanvia El-Habiby ant Prof. Dr. Rizk El-Bealy Texhle Department. Faculty of Engineering. Hansoura linversity:

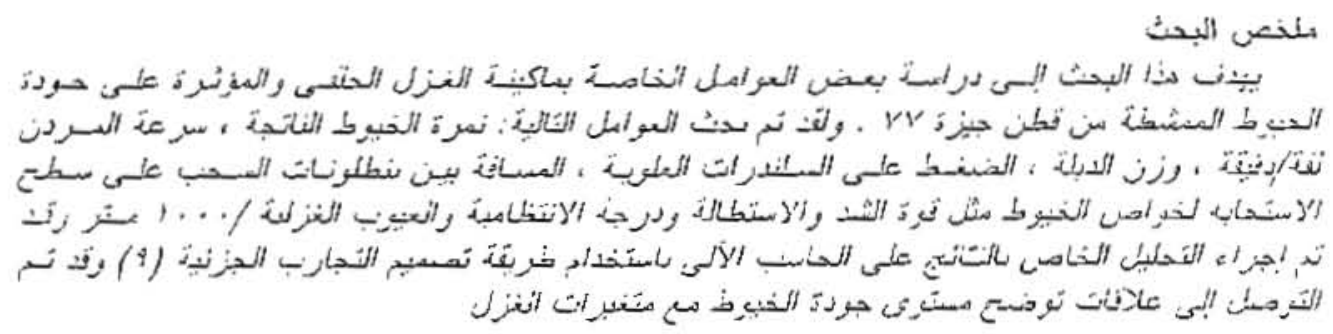

\section{Abstract}

In the present work a study was made about the effect of the following five parameters' 'yam linear density, spindle speed, traveller size, top roller pressurc and cradle opening" on the quality of ring spun yarn.

The expcriments were carried out by varymg each parameter at two ievels using the fractional factorial tcchuque (9). The experimental design treatunent with the help of mini-computer programming was ased to detenune the effect of man paraineters and two factor interactions. A statistical analysis for the study of the sıgnificance of inain variables and interactions between iwo parameters were carreci out by the method of variance anaiysis.

\section{I- Introduction:}

it is well known that spiuning performance and yarn quality at rung spirming frame are governed by many factors, such as, roller eccentrecity, spindlc speed, travelier weight, top roller loading, cradie spacing. break draft, drafting roller setting, feeding conditions and twist of the input roving $(14,16,17,5,8)$

$R$. Audivert et al. (13) studied the effect of apron spacing on yarn quality using four values of spacing and stated that : increase in apron spacing was tumed mto decreasing in boih tenacity and unifonmity of ring spun yams. End breaks also have been shown to increase as apron spacing increases $(12,17)$.

Yabionskii (11) studied the effect of roller wemghting on yan quality and end breakage ratc and found that. there was a high negative correlation between weighing of the delivery rollers and the average number of end breaks. However no significant correlation was found between delivery rollers weighing and yan properties.

Audiven et. al (7) studied the effect of draftug speed on yarn irregularity. It was fout that . Increasing the drafting speed in ieruns of spindle specd increase: 
yarn irrcgularity when fibres of high extensibility and low modulus were employed. Conversely, increasing drafting speed improves regularity of yams spun from ribres of low extensibility and high modulus.

Earlier working on high draft ring frames equipped witl casablanca aproi cradles; Simpson et. al. $(2,3,4)$ found that, spinung draft has more effect upon yan strength and yam uniformity than other parameters. The optimum draft value was found to vary with yarn count and cotton tibre parameters.

An earlier study $(14)$ indicated that the hank of the imput roving and ring frame draft can be varied over a wide range in top arm loading without affecting yarn quality in coarse and medium varieties of cotton.

Simpson et. al.(1) studied the effect of doubling at ring frame, they found that, the benefits of doubling depend upon the count spun as well as the total draft. Also Rantnam (16) conciuded that, there is a little difference between the quality of yam when using single and double feeds in counts 40 s and above .

Earlier work on top arm drafting Salasubramanian. (14) have shown that, yam quality is also affected by the input hank and feeding conditions (single or double). Further studies indicated a high degree of interaction berween the different processing variables, and the optimum with respect to each of them is found dependent upon the value of the parameters.

El-Bealy et. al. (18) studied the influence of drafting zone parameters, such as: break draft, distance clips, total draft, drafting spezd and blend properties at the ring frame on the quality of two-component blended yarn. The investigation was carried ont by varying each two varıables at different levels while the others were kept constant.

E1-Behery (15) cartied out methodolgy studies to determine the effects of machine parameters, break draft, spacer stud and total draft on spiuning end breakage, strength, elongation and yarn irregularity for two types of cotton spun on the Sacoloweil Magna-draft system for both short and long cradle types.

Thus the present study was ained to investigate the effect of yam linear density,, spindle speed, traveller number, top roller pressure and cradle spacing using fractional factorial design technique to get a proper understanding of their effect as individuals or interactions on yarn tenacity, unformiy and yarn imperfections

\section{2- Experimental work:}

\section{2-1 Material uset:}

Egyptian cotton fibres $G_{77}$ of $32 \mathrm{~mm}$ mean length, 3.81 Micronair reading and pressely index $11.1(1 \mathrm{~b} / \mathrm{mg})$ and trash content $3.64 \%$ was used in the present study.

\section{2-2 Yarn production}

The machine sequence was adopted for cotton fibre as shown in Fig.(1)(ail items were put through). 


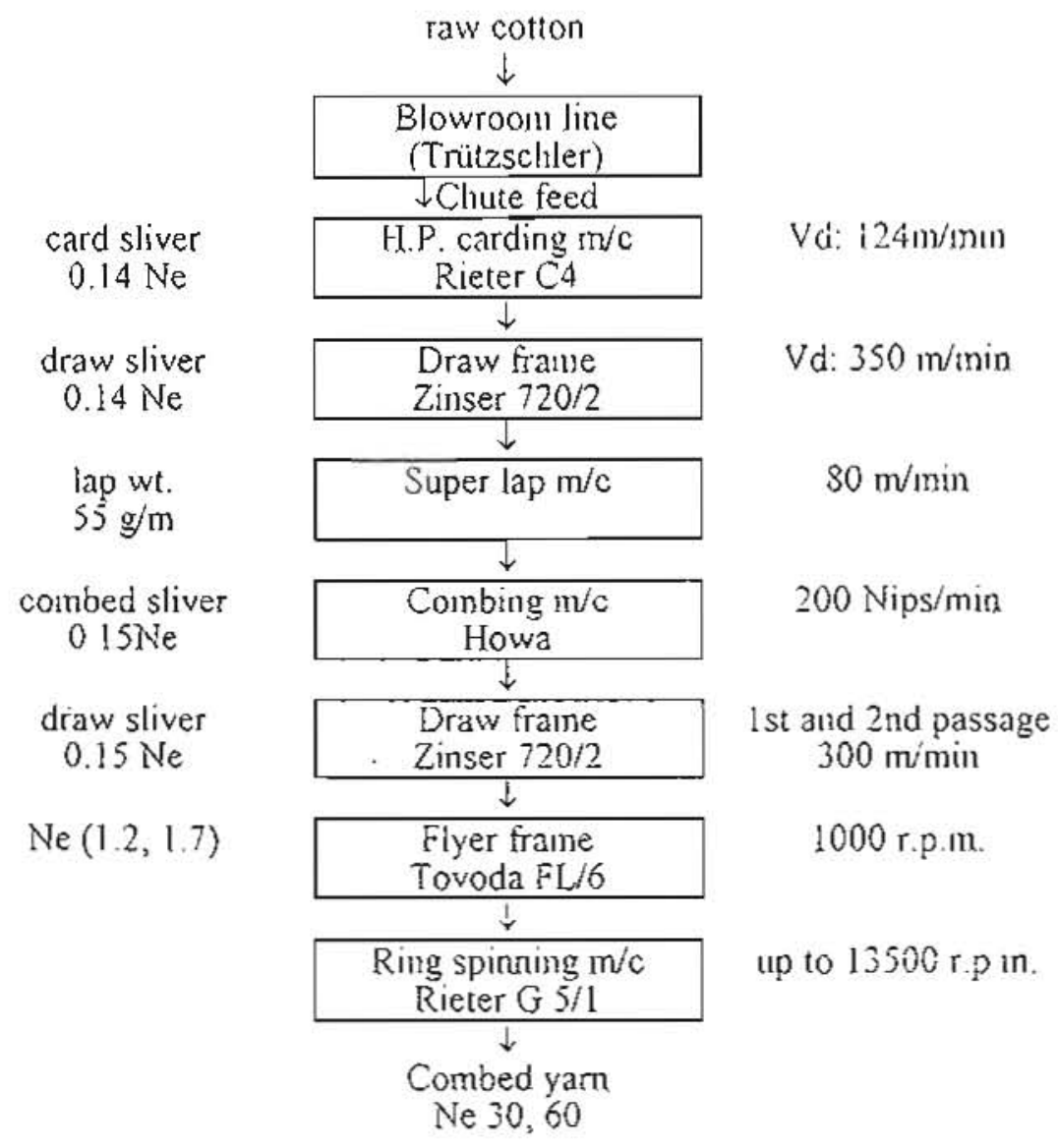

\section{$V d$ : delivery speed}

Fig. (l) Machine Sequence

\section{2-3 Measurements:}

Yarn Evenness: for each test 10 bobbins were presented to Uster Evenness Tester It. Material speed was $200 \mathrm{~m} . / \mathrm{min}$, with testing ume $2.5 \mathrm{~min}$. The yarn nep counts, thin places and thick places were tested under the same conditions.

Yanı strength: Tests were performed on Uster Tensomat.Batches of 10 bobbins were tested with 20 indindual tests / bobbin.

The count, and count variation of the fulal ring yam in C.V\% was tested usmg Uster Autosorter.

Fibre parameters were examined for length characteristics on Digital Fibrograph, trash content on shirley analyser and fineness on Shiefeld Micronaır. 


\section{2-4. Statistical Design:}

The general method of analysis of the present study which is drawn from the previous literature of experimental design $(9)$ wiil be described here. The fractional factorial experinents was set up in order to investigate the quality of ring spin yan and to optimize the yam linear density, spindle speed (r.p.m), (raveller wcight (n:mber), loading the top arm $(\mathrm{Kp})$ and cradle spacing $(\mathrm{mm})$.

The investigation of ring variables each at two leveis will entait 32 observation $\left(2^{5}\right)$, but the aim of the present investigation is to obtain information on main effect and interaction with the technique of "five factors in sixteen observation" | |han is required by the complete design as shown in Tabie (2).

Table (1) Levels of Variables

\begin{tabular}{|l|c|c|c|c|c|}
\hline \multirow{2}{*}{ Levels } & \multicolumn{5}{|c|}{ Variables } \\
\cline { 2 - 6 } & $\mathrm{X} 1$ & $\mathrm{X} 2$ & $\mathrm{X} 3$ & $\mathrm{X} 4$ & $\mathrm{X} 5$ \\
\hline & $\mathrm{Ne}$ & r.p.m & tr. no. & $\mathrm{kp}$ & $\mathrm{mm}$ \\
\hline Lower level $(-)$ & 30 & 9000 & $2 / 0$ & 15 & 2.5 \\
\hline Upper level $(+)$ & 60 & 13500 & $5 / 0$ & 18 & 4.0 \\
\hline
\end{tabular}

Table (2) Plan of Experiments

\begin{tabular}{|c|c|c|c|c|c|c|}
\hline \multirow{2}{*}{$\begin{array}{c}\text { Combination } \\
\text { No. }\end{array}$} & \multicolumn{7}{|c|}{ Level of Variables } \\
\cline { 2 - 7 } & $\mathrm{X} 1$ & $\mathrm{X} 2$ & $\mathrm{X} 3$ & $\mathrm{X} 4$ & $\mathrm{X} 5$ & Response \\
\hline 1 & - & - & - & - & - & $\mathrm{y} 1$ \\
2 & + & - & - & - & + & $\mathrm{y} 2$ \\
3 & - & + & - & - & + & $\mathrm{y} 3$ \\
4 & + & + & - & - & - & $\mathrm{y} 4$ \\
5 & - & - & + & - & + & $\mathrm{y} 5$ \\
6 & + & - & + & - & - & $\mathrm{y} 6$ \\
7 & - & + & + & - & - & $\mathrm{y} 7$ \\
8 & + & + & + & - & + & $\mathrm{y} 8$ \\
9 & - & - & - & + & + & $\mathrm{y} 9$ \\
10 & + & - & - & + & - & $\mathrm{y} 10$ \\
11 & - & + & - & + & - & $\mathrm{y} 11$ \\
12 & + & + & - & + & + & $\mathrm{y} 12$ \\
13 & - & - & + & + & - & $\mathrm{y} 13$ \\
14 & + & - & + & + & + & $\mathrm{y} 14$ \\
15 & - & + & + & + & + & $\mathrm{y} 15$ \\
16 & + & + & + & + & - & $\mathrm{y} 16$ \\
\hline
\end{tabular}


Table (3) Yarn properties

\begin{tabular}{|c|c|c|c|c|c|c|c|}
\hline \multicolumn{8}{|c|}{ Yarn Properties } \\
\hline \multirow{2}{*}{$\begin{array}{c}\text { Cumbination } \\
\text { No. }\end{array}$} & \multirow{2}{*}{$\begin{array}{c}\text { Strength } \\
\text { g. }\end{array}$} & \multirow{2}{*}{$\begin{array}{l}\text { Tenacily } \\
\text { g./tex }\end{array}$} & \multirow{2}{*}{$\begin{array}{c}\text { Elangation } \\
\%\end{array}$} & \multirow{2}{*}{$\begin{array}{c}\text { Irregularity } \\
\text { C. V\% }\end{array}$} & \multicolumn{3}{|c|}{ limperfectiunss $1000 \mathrm{in}$} \\
\hline & & & & & thin & lhick & neps \\
\hline 1 & 450.74 & 22.88 & 5.42 & 910 & 0 & 2.3 & of \\
\hline 2 & 205.47 & 20.86 & 3.77 & 1363 & 12.7 & 34.5 & 60.6 \\
\hline 3 & 436.55 & 22.16 & 4.6 & 9.40 & 0 & 2.2 & 14 \\
\hline 4 & 207.44 & 21.06 & 2.15 & 13.49 & 15.1 & 24.7 & 61.4 \\
\hline 5 & 430.25 & 21.84 & 536 & 9.11 & 0 & 2.2 & 12 \\
\hline 6 & 210.30 & 21.35 & 393 & 12.48 & 34 & 16.9 & 51.5 \\
\hline 7 & 434.98 & 22.08 & 4.57 & 9.09 & 0 & 1.7 & 9.8 \\
\hline 8 & 204.58 & 2077 & 3.40 & 13.30 & 168 & 233 & 72.8 \\
\hline 9 & 46531 & 23.62 & 5.62 & 892 & 0 & 22 & 12.1 \\
\hline 10 & 200.84 & 2039 & 3.59 & 12.25 & 4.3 & 12.3 & 51.9 \\
\hline 11 & 456.45 & 23.17 & 4.74 & 8.82 & 0.1 & 2.5 & 12.0 \\
\hline 12 & 190.89 & 1938 & 2.49 & 14.05 & 24.1 & 26.6 & 70.0 \\
\hline 13 & 471.03 & 23.91 & 535 & 8.94 & 0.2 & 2.4 & 13.2 \\
\hline 14 & 21355 & 21.68 & 4.18 & 12.97 & 10.1 & 196 & 55 \\
\hline is & 436.75 & 22.17 & 464 & 9.19 & 01 & 2.3 & 14.8 \\
\hline 16 & 208.03 & $2 ! .12$ & 3.57 & 12.44 & 7.9 & 16.7 & 56.4 \\
\hline
\end{tabular}

Table (4)

Regression coefficients

\begin{tabular}{|c|c|c|c|c|c|c|c|}
\hline \multirow{3}{*}{$\begin{array}{l}\text { Regtession } \\
\text { coefficient }\end{array}$} & \multicolumn{7}{|c|}{ Responce Parameter } \\
\hline & \multirow{2}{*}{$\begin{array}{c}\text { Strength } \\
\mathrm{g} .\end{array}$} & \multirow{2}{*}{$\begin{array}{c}\text { Tenacity } \\
\text { g/tex }\end{array}$} & \multirow{2}{*}{$\begin{array}{c}\text { Elangation } \\
\%\end{array}$} & \multirow{2}{*}{$\begin{array}{c}\text { Irregularity } \\
\text { C. V\% }\end{array}$} & \multicolumn{3}{|c|}{ Imperfections/1000m } \\
\hline & & & & & thin & thick & neps \\
\hline bo & 310.33 & $21 . \overline{781}$ & 4.21 & 1107 & 5.925 & 12.025 & 36.063 \\
\hline bl & -108.76 & -0953 & -0.804 & 2002 & 5.875 & 9.782 & 23.887 \\
\hline b2 & -621 & -0.291 & 0.441 & -0.149 & 2.088 & 0.475 & 2.838 \\
\hline b3 & 0.85 & 0.088 & 0.164 & -0134 & -0.113 & -1.388 & -0.375 \\
\hline 64 & 2.45 & 0.52 & 0.061 & .0126 & -0125 & -1.45 & -0.388 \\
\hline bs & -4.86 & 0.744 & 0.048 & 0.248 & -2.05 & -2.088 & -2.85 \\
\hline b12 & 2.46 & 0.047 & -0.042 & 0.95 & 2.088 & 0.525 & 2.363 \\
\hline b13 & 4.396 & 0.314 & $022 \mathrm{I}$ & -0.145 & 0.188 & -1.313 & -0.65 \\
\hline b/4 & -3.83 & .0334 & 0.012 & -0024 & -0.125 & -1.588 & -1.238 \\
\hline b15 & 3.23 & 0.061 & -0.028 & 0.164 & -2.075 & -2.088 & -1.8 \\
\hline b23 & $\cdot 0.92$ & -0.043 & 0109 & -0.084 & -0.7 & -0.113 & 0.075 \\
\hline b24 & -3.52 & -0.179 & 0.029 & 0.028 & 0.113 & 0975 & -0.213 \\
\hline b2s & -1.334 & 0.153 & 0.034 & 0.015 & 138 & 0.988 & -1.15 \\
\hline b34 & 0.915 & 0.199 & -0.001 & 0.075 & -0.163 & 1.063 & -0.45 \\
\hline b35 & -1.77 & 0.031 & 0.026 & -0.045 & 0113 & 0.875 & -0.113 \\
\hline 645 & -1.046 & 0.002 & 0.086 & 0.088 & -0.675 & -0.013 & 0.55 \\
\hline
\end{tabular}


Table (5-a)

Summary of Variance Analysis

\begin{tabular}{|c|c|c|c|c|c|c|c|c|}
\hline \multirow{4}{*}{$\begin{array}{c}\text { Source } \\
\text { of } \\
\text { Variance }\end{array}$} & \multirow{4}{*}{$\begin{array}{c}\text { Degrce } \\
\text { of } \\
\text { Frecdom }\end{array}$} & \multicolumn{7}{|c|}{ Mcan Square (M S) } \\
\hline & & \multicolumn{7}{|c|}{ Yam Properties } \\
\hline & & Strength & Tcnacity & Elongation & |3rregularits & Imperfe & ections f & $1000 \mathrm{~m}$ \\
\hline & & $g$ & $g / t c x$ & $\%$ & C.V\% & thin & nck & neps \\
\hline \multicolumn{9}{|l|}{ (!) Main effects } \\
\hline Yarı count ( $\mathrm{Ne}) \mathrm{XI}$ & 1 & 189279 & 14.53 & 10336 & 64.12 & $\$ 52.25$ & $153 \times 6$ & $9129 \mathrm{x}$ \\
\hline Spundic Specd (r p.m) $X_{2}$ & ! & 616.9 & 1.35 & 3.115 & 0.35 & 69.72 & 361 & 129.39 \\
\hline Traveller number X3 & 1 & 11.65 & 0.1278 & 0429 & (1) 29 & 19.80 & 3080 & 2.25 \\
\hline Top coiler pressureXt & 1 & 9629 & 0.36 .3 & $0(1600$ & () 26 & 0.20 .3 & 3364 & 2.40 \\
\hline Cradle spacing Xs & 1 & 37782 & 0.74370 & $0,0.37$ & 097 & 6.4 .80 & (69) 72 & 129.96 \\
\hline \multirow{2}{*}{\multicolumn{9}{|c|}{$\begin{array}{l}\text { (ii) Two-Factor } \\
\text { Interacions }\end{array}$}} \\
\hline & & & & & & & & \\
\hline $\mathrm{x} 1 \times 2$ & $i$ & 8677 & 01135 & 04360 & $\cap 1+4$ & 69) 72 & $4+1$ & 89) 30 \\
\hline $\mathrm{X} 1 \times 3$ & 1 & $3(x) ; 2$ & 1.5805 & 07863 & 0.3 .36 & 20.70 & 27.56 & 0.20 \\
\hline$X I X 4$ & 1 & $234+7$ & $17 \times 91$ & 0.0020 & $000 \%$ & 0.25 & 400 & : : \\
\hline XIXS & 1 & 167.38 & 0.0613 & 00125 & 0429 & 68.89 & 6972 & 484 \\
\hline$\times 2 \times 3$ & 1 & 13.59 & 00297 & 0.1911 & 0.112 & 7.84 & 2916 & 6.76 \\
\hline$\times 2 \times 4$ & 1 & 198.46 & $05(49$ & $0(11,32$ & 0,013 & 203 & 15.21 & 2450 \\
\hline$\times 2 \times 5$ & 1 & $28+9$ & $0 \$ 750$ & 0.0187 & 0.004 & 0.276 & $15.60)$ & 5184 \\
\hline$x 3 \times 4$ & 1 & 1341 & 06362 & 0.00003 & 0.081 & 0.423 & 1806 & 0,09 \\
\hline Xixs & 1 & 50.23 & 00149 & 00110 & 0.032 & $0.20=$ & 12.25 & 0.723 \\
\hline$x 4 \times 5$ & 1 & 17.54 & 0,0005 & 0.1198 & 0123 & 729 & 7064 & 21.16 \\
\hline
\end{tabular}

(For $n=10) \quad\left({ }^{*}\right)$ Significance for $99 \% \quad\left({ }^{* *}\right)$ Significance for $95 \%$

$\left({ }^{* * *}\right)$ Significance for $90 \%$

The five variabies mentioned will be refered to as $X 1, X 2, X 3, X 4$ and $X 5$ respectively. The levels chosen for each varnable as shown in Table (1), considering the previous practical experience(9)

\section{3-Results and Discussion}

The results of yari quality tests at different conditions "16 experimentai combination" for yan count, spindie speed, treaveller weight, top roller pressure and cradle spacing are shown $n$ Table (3). Regression coefficients were detennined for the measured yarn quality and tested for significance are given in Table (4) 
Sumnary of variance analysis of different yarn characieristucs is shown in Tabic (5a) and $(5-b)$. Also two factor intcractıons whinch kave a signuficant effect on yan characteristics are given in Tables(6).

Table (5-b)

Summary of Variance Analysis

\begin{tabular}{|c|c|c|c|c|c|c|c|c|}
\hline \multirow{4}{*}{$\begin{array}{c}\text { Source } \\
\text { of } \\
\text { Vartance }\end{array}$} & \multirow{4}{*}{$\begin{array}{c}\text { Degree } \\
\text { of } \\
\text { Frecdom }\end{array}$} & \multicolumn{7}{|c|}{ Mcans Squarc (M.S) } \\
\hline & & \multicolumn{7}{|c|}{ Yam Propertics } \\
\hline & & \multirow{2}{*}{$\begin{array}{c}\text { Strength } \\
2\end{array}$} & \multirow{2}{*}{$\begin{array}{c}\text { Tcnacity } \\
\text { g/tex }\end{array}$} & \multirow{2}{*}{$\begin{array}{c}\text { Elongation } \\
\%\end{array}$} & \multirow{2}{*}{$\begin{array}{c}\text { Irrcguiarity } \\
\text { C V\% }\end{array}$} & \multicolumn{3}{|c|}{ Imperfections/ $1000 \mathrm{~m}$} \\
\hline & & & & & & thin & thick & neps \\
\hline \multicolumn{9}{|l|}{ (I) Main effects } \\
\hline Yarn couru (Ne)XI & 1 & 1892794 & 14.53 & 10336 & 6412 & $\$ 52,25$ & 15386 & $\% 12 \% \mathrm{~s}$ \\
\hline Spindile Speed (r.p ni) X2 & 1 & 6169 & 1.35 & 3115 & 0.35 & 69) 72 & 3.61 & $12 \% .39$ \\
\hline Traveiler number $X$ ר & 1 & 1165 & 0.1278 & 0.429 & 029 & 1980 & 30811 & 2.25 \\
\hline Top rollet pressurc $X 4$ & 1 & 9629 & 0363 & 0.060 & 0.26 & 020.3 & 3364 & 240 \\
\hline Cradlc spacing $X 5$ & 1 & $\$ 77.82$ & 0.74170 & 0037 & 0.97 & 6480 & 61) 72 & 12996 \\
\hline \multirow{2}{*}{\multicolumn{9}{|c|}{$\begin{array}{l}\text { (ii) Two-Factor } \\
\text { Interacions }\end{array}$}} \\
\hline & & & & & & & & \\
\hline$x 1 \times 2$ & 1 & 96.77 & 0035 & $0+360$ & 0.144 & 69.72 & +41 & si) $: 0$ \\
\hline$x: x_{3}$ & 1 & 30932 & 15805 & 67863 & 0336 & 2070 & 27.56 & (0) 201 \\
\hline Xixt & 1 & $2.34+7$ & 1.7891 & 0.0020 & $(100)$ & 0.25 & 30.0 & 3.34 \\
\hline XIX5 & 1 & 16738 & 00613 & 0.0125 & 0.429 & 6880 & 6972 & $4 \times 4$ \\
\hline $\mathrm{X} 2 \mathrm{X} 3$ & 1 & 1359 & $0: 297$ & 0.1911 & 0112 & $7 \$ 4$ & 29.16 & 676 \\
\hline$\times 2 \times 4$ & $!$ & 198.46 & $0 \leqslant 149$ & 00132 & 0.013 & 20,3 & 1521 & $2+50$ \\
\hline$\times 2 \times 5$ & 1 & $28+9$ & 0.5750 & 00187 & 0004 & 0.276 & $15(6)$ & 51,84 \\
\hline$x .3 \times 4$ & 1 & $13,+1$ & 06362 & 006003 & 0.081 & $0+23$ & 1806 & $0(10)$ \\
\hline$x \leqslant \times 5$ & 1 & 5023 & 00149 & 0,0110 & 0032 & 0203 & 12.25 & (1) 72 ; \\
\hline$\times 4 \times 5$ & 1 & 1754 & 0.00095 & 01198 & 123 & 7.29 & 7064 & 2116 \\
\hline
\end{tabular}

(For $n=6$ ) $\quad\left({ }^{*}\right)$ Significance for $99 \% \quad\left({ }^{* *}\right)$ Significance for $95 \%$ $\left({ }^{* * *}\right)$ Significance for $90 \%$

\subsection{Yarn Strength}

The analysis of results are given in Tabies $(5-a)$ and $(5-b)$, the inain effect of individual five paraneters and two-factor interactions wiule three fnctor which are regardert as negigibie. The stgnificant effects m order of magnitude are X1: yarn count, $X 2$ spundle speed and $X 5$ - spacer setting From the variance analysis, it can be noticed that yam count, spundle speed and cradle spacing have a significant cffect 
(i) Yarn Strength

Table (6) Tivo- Factor Interaction

* Single end strength (gm)

inicracion $\mathrm{X}, \mathrm{X}_{3}$;

\begin{tabular}{|c|cc|}
\hline$X_{1}$ & \multicolumn{2}{|c|}{$X_{3}$} \\
\hline$*$ & +232 & +16.1 \\
+ & 1969 & 2074 \\
\hline
\end{tabular}

Interaction $X_{1} X_{a}$

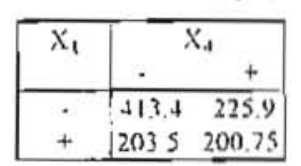

* Tenacity (g/tex)

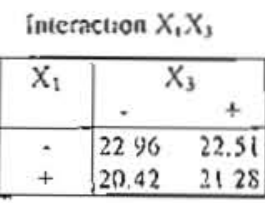

Interacion $X_{1}, X_{3}$

\begin{tabular}{|c|c|c|}
\hline \multirow[t]{2}{*}{$x_{1}$} & \multicolumn{2}{|c|}{$\overline{\bar{x}_{s}}$} \\
\hline & . & + \\
\hline - & 427.8 & 4116 \\
\hline$\div$ & 203.8 & 200.5 \\
\hline
\end{tabular}

Imeraction $X_{1} X_{\text {e }}$

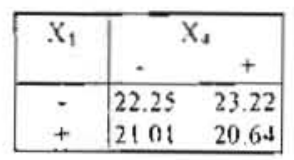

(ii) Yarn Elongation

Ineracion $X_{1} X_{1}$
\begin{tabular}{|c|lc|}
\hline$X_{1}$ & \multicolumn{2}{|c|}{$X_{3}$} \\
&. & + \\
\hline$:$ & 5.095 & 498 \\
+ & 30 & 3.77 \\
\hline
\end{tabular}

(iii) Yarn Irregularity

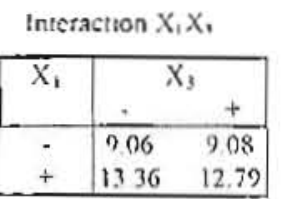

Ineraction $X_{1}, X_{5}$

(iv) Yarn Imperfections

\section{* Neps $/ 1000 \mathrm{~m}$}

Interaction $X_{1} X_{2}$

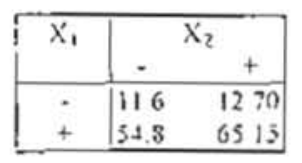

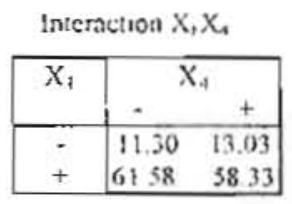

*Thin places $/ 1000 \mathrm{~m}$.

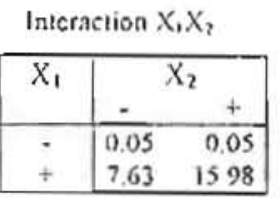

* Thick places $/ 1000 \mathrm{~m}$.

\begin{tabular}{|c|c|c|}
\hline \multirow[t]{2}{*}{$\overline{X_{1}}$} & \multicolumn{2}{|c|}{$x_{3}$} \\
\hline & - & + \\
\hline- & 2.23 & 2.23 \\
\hline+ & 17.65 & 260 \\
\hline
\end{tabular}

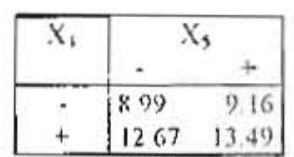

tuteracion $X_{1} X_{5}$

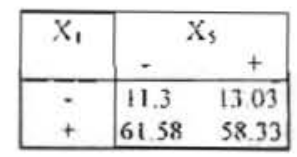

lacenction $X_{:} X_{6}$

\begin{tabular}{|c|cc|}
\hline$X_{2}$ & \multicolumn{2}{|c|}{$X_{5}+$} \\
\hline+ & 31.53 & $34+3$ \\
+ & 34.9 & 42.9 \\
\hline
\end{tabular}

Interacion $X_{1} X_{3}$

\begin{tabular}{|c|cc|}
\hline$X_{1}$ & \multicolumn{2}{|c|}{$X_{3}$} \\
\hline & - & + \\
\hline$*$ & 0075 & 0.025 \\
+ & 7.68 & 1593 \\
\hline
\end{tabular}

Inictaction $\mathrm{X}: \mathrm{X}$,

\begin{tabular}{|c|cc|}
\hline$X_{4}$ & \multicolumn{2}{|c|}{$X_{S}$} \\
\hline- & 11.4 & + \\
\hline+ & 8.48 & 12.56 \\
\hline
\end{tabular}


on yam strength propenty" single end strength, tenaciiy and elongatton". Yam Icnacity decreases as spundle speed increases from 9000 to 13500 r.p. m as shown in Fig $(2-1)$. These results are in agreement with earlier work (10). This could be explamed on the basis of mcreased drafting speed, increases the average fibre rension which resulung in an increase in the fibres dragging out of the roving unto the front roiler nip and this heips in reducing yam strength.

In the present anaiysis, it is considered that the two factor interactions (10 items) were combined to give an estimate of error variance as shown in table $(5-1)$. Also since the interaction of X1X3, XIX4, XIX5 and X2X4 are large, the six interactons were combined to give an estimate of error varsance as shown in Table (5-b). Thius it is clear that from the statistical analysis, the two factor interaction XIX3 yan: count with traveller weight; XIX4 yarn count with loading the top arm affect significantly at $95 \%$ level as shown in Table (5-a and 5-b) Whitc the interaction of XIX5 yan count with cradle spacing and X2X4 spindle speed wilh pressure have a slight influence on yan strength.

From the two way tabies (5) and (6) and Fig (4), it cun be seen that the yam expected count has a significant effect on yan strength, whlutever, the conditions of the other factors (traveller weight, loading the top anm). There is a high value of yan strength obtaned at low level of yan count (i.e for coarse count). Also the traveller weight effect has been notuced, the lugh traveller number especiaily at higher tevel of yan count" fine count" resultung in a lower yan strength than those obtaned for coarse count. For the experimental yarn counts $(30$ to $60 \mathrm{Ne}$ ), mcreasing loading the top arn restited in a lower yan strength. Also closer setting witl) course or fine counts results in a higier strength than that obtained at wide spacer, this is due to better control of fibres by closer cradle setting. On the oher hand, the effect of spindle spced with pressure at top roller can be noticed in Table (6). The results indicate that a higher yan strength at lower level of spindle speed (for the two leveis of top roller pressure) than those obtained for higher levet of spindle speed.

\subsection{Yarn elognation:}

From the expermental results of yam elongation [Tables $(5-a)$ and $(5-b)$ ], it can be seen that, yan count and spindle speed have a sigmincant effect on yam elongatıon. Higher spundle speed has a negatıve effect on yan elongatıon as shown in Fig (2-2).

The influence of two factor interaction on yarn eiongation is given wa Fig. (5) and Tabie (6) The mteractuon between yam count and traveller weight has a significant influence on elongation at $95 \%$ confidence level. Also from two way Tabies (6) and Fig (5-2), tt is cleat that, as yan gets funer ts elongation is largly decreased by using heavy traveller weight. 


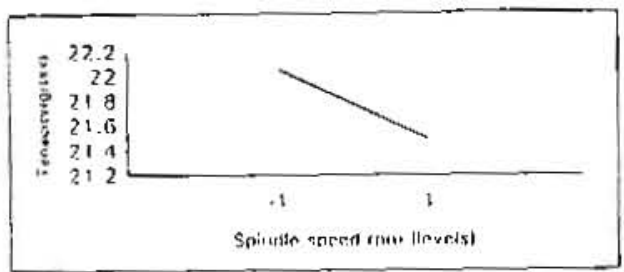

$(2-1)$

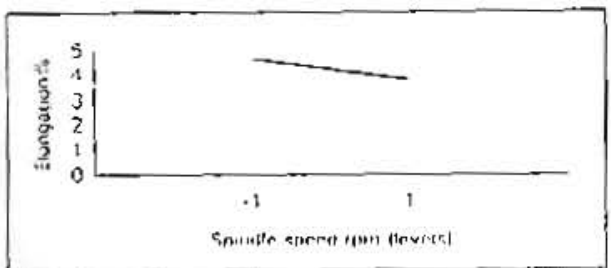

$(2-2)$

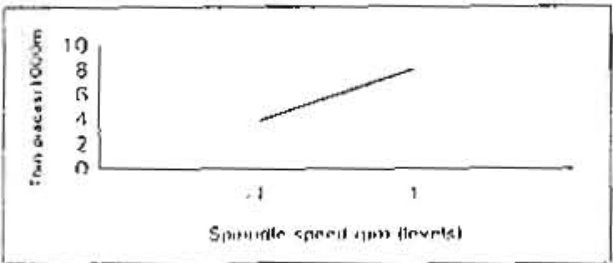

$(2-3)$

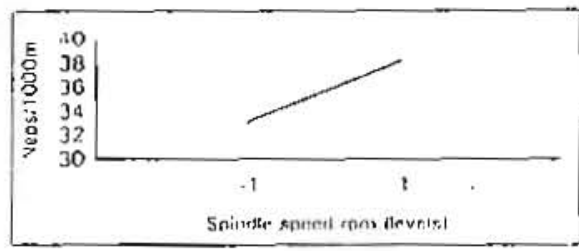

$(2-4)$

Fig. (2)

Relation Between Spindle Speed and Yarn Properties
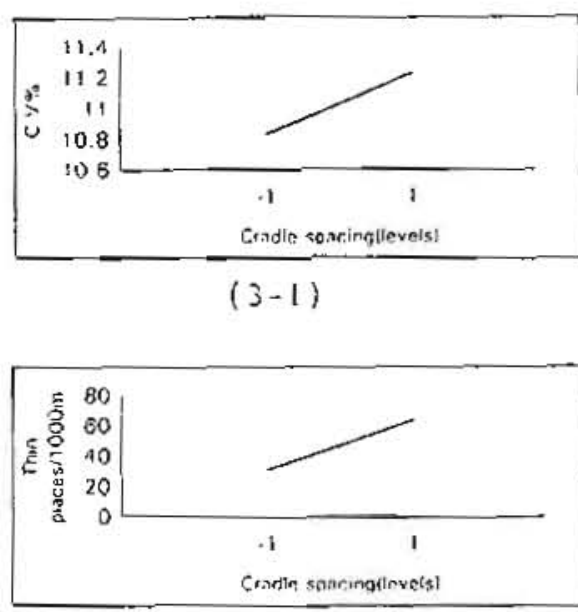

$(3-2)$

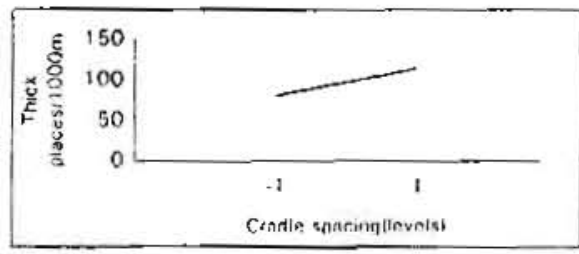

$(3-3)$

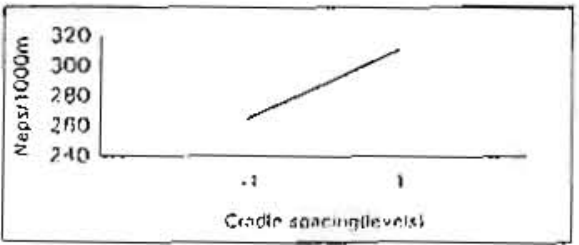

$(3-4)$

Fig. (3)

Relation Between Cradle Spacing and Yarn Properties 

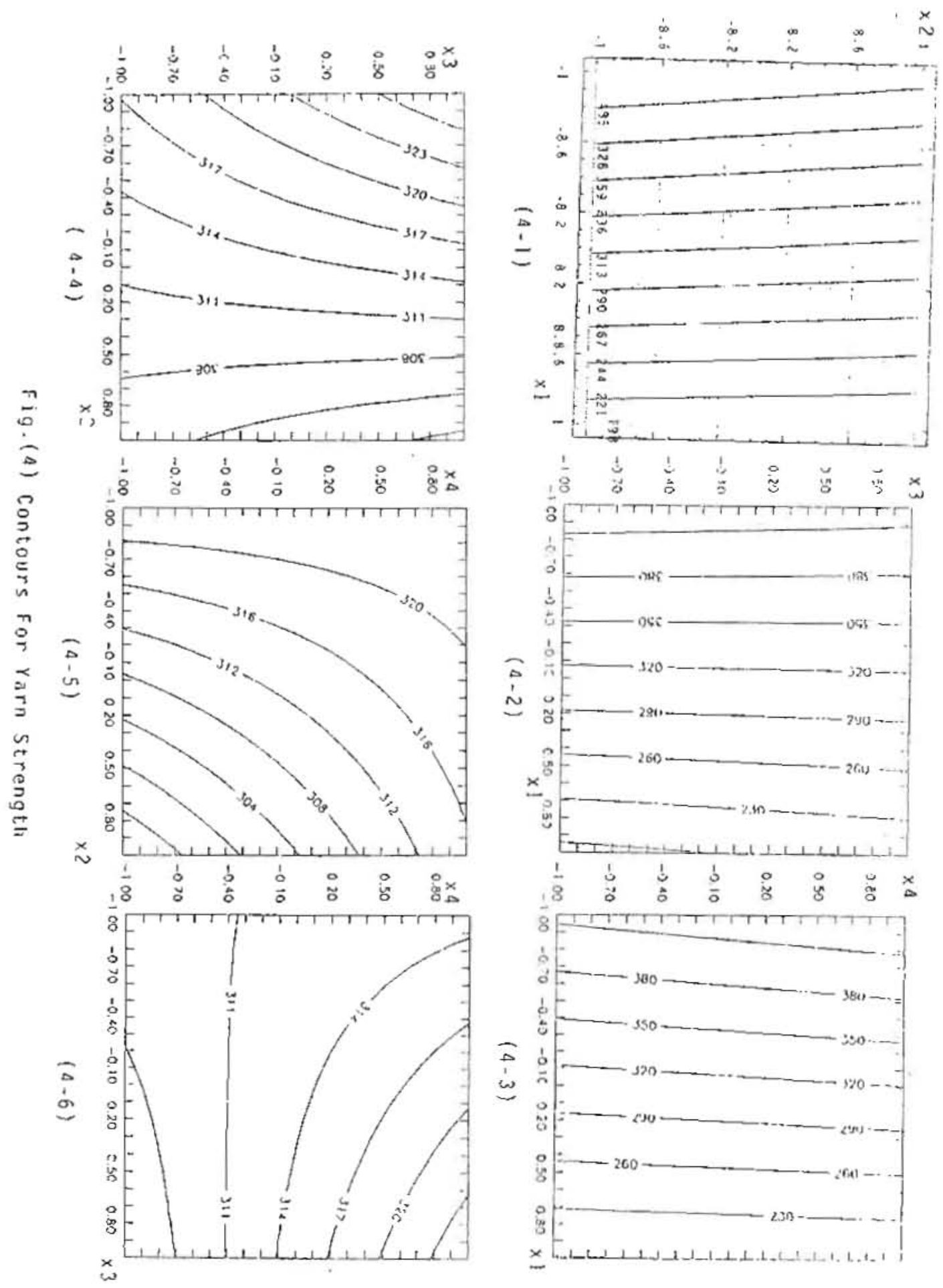

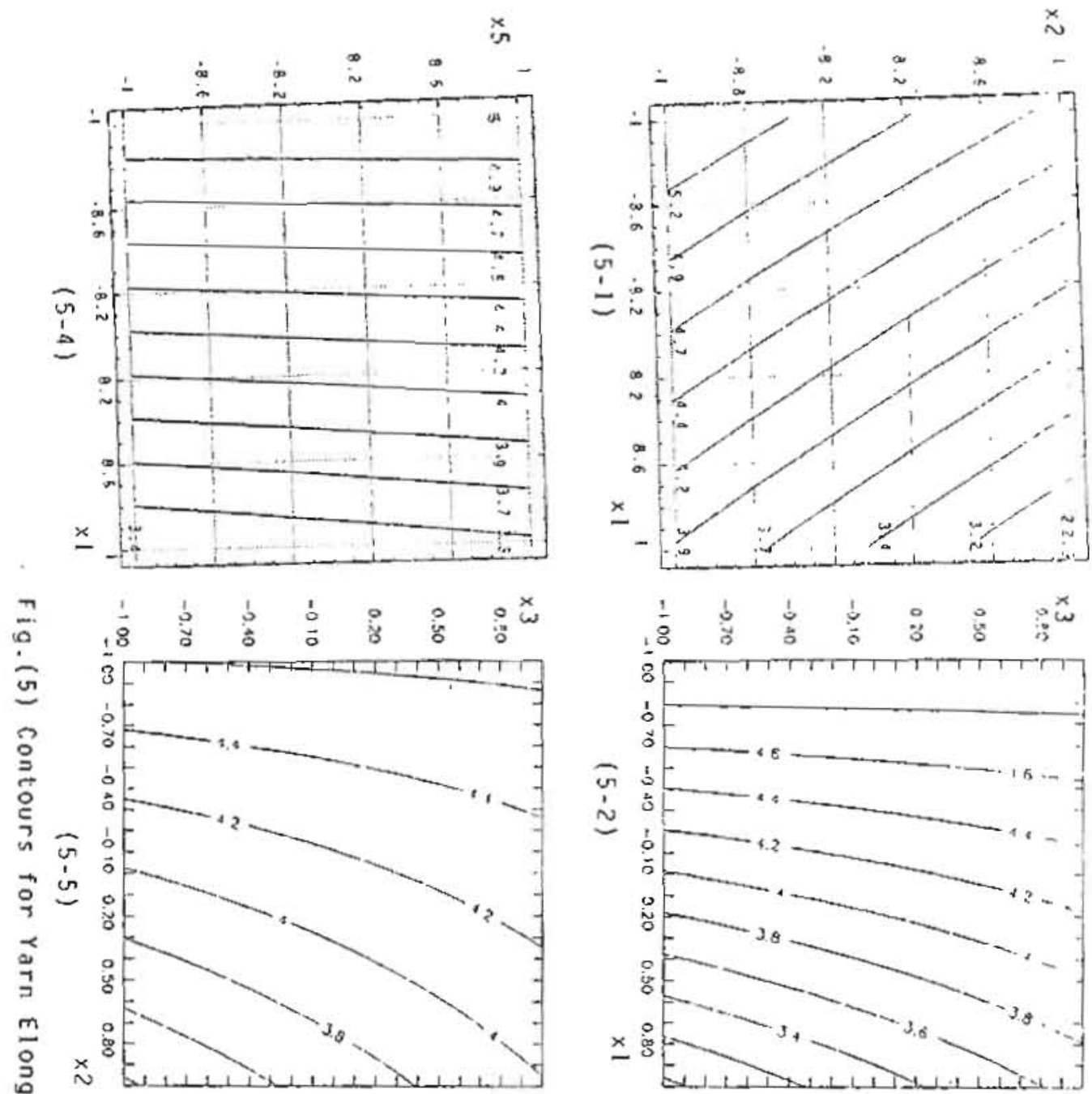

혹
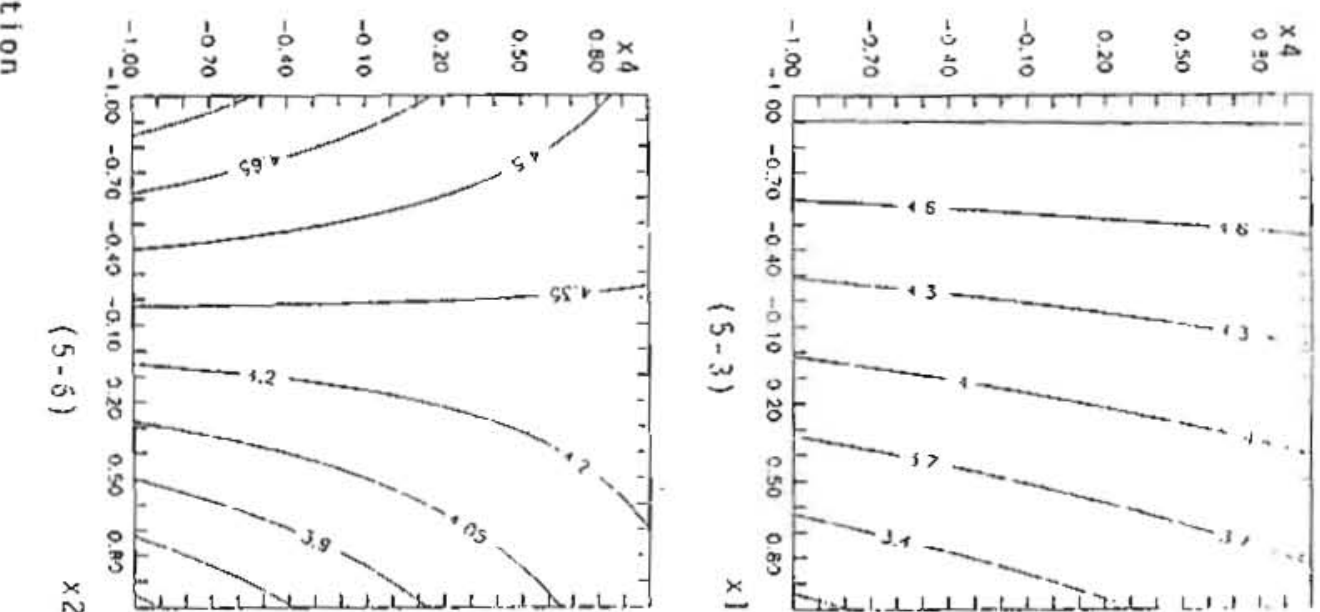
Mansoura Engineering Joumal (ME.), Vol. 21. No. t. December $1996 \quad$ 7:13
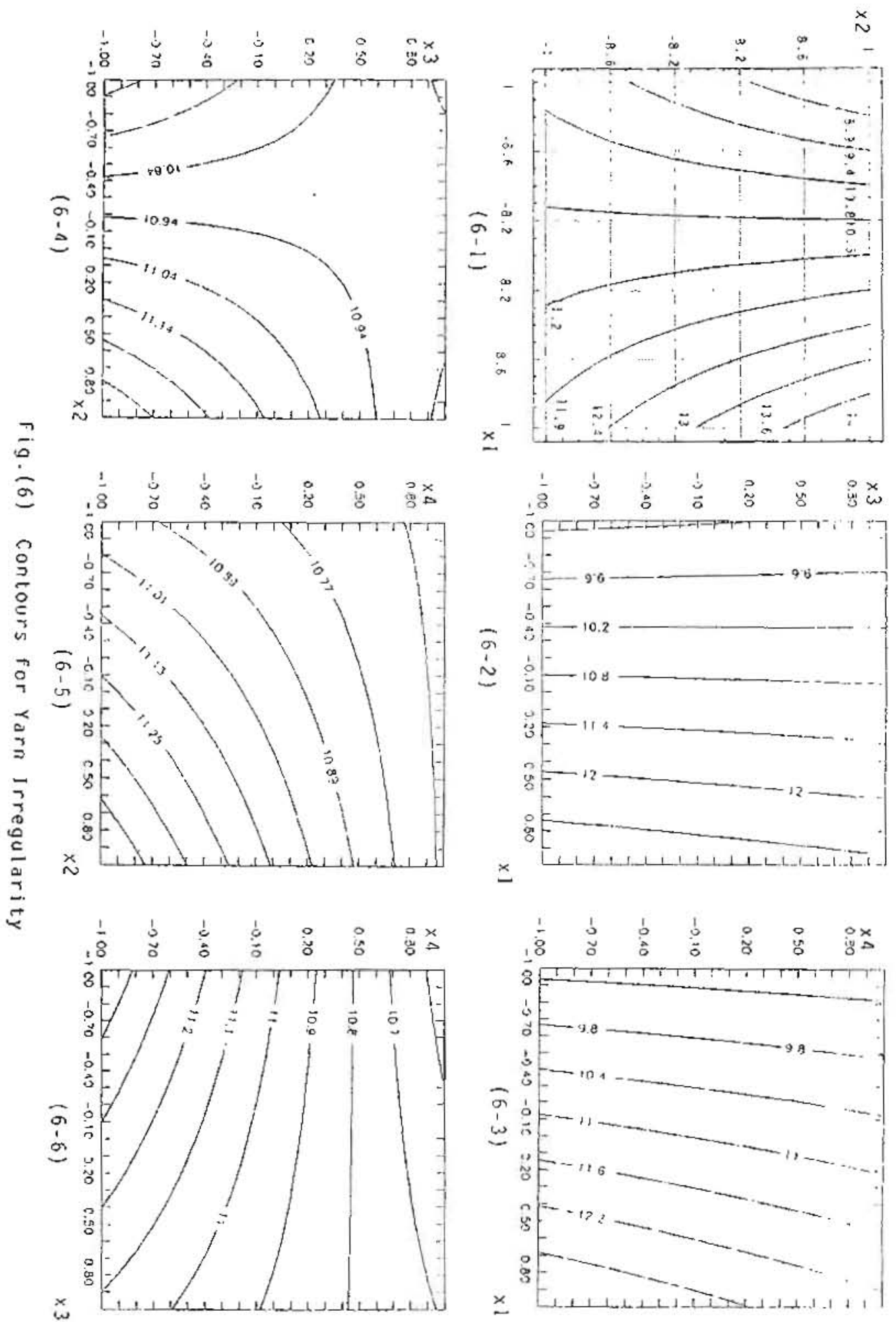

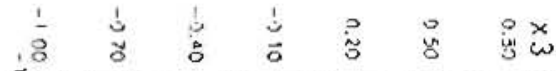

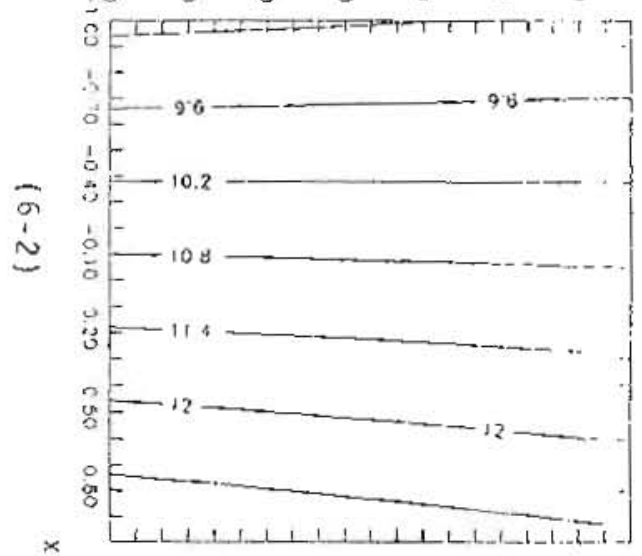

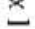

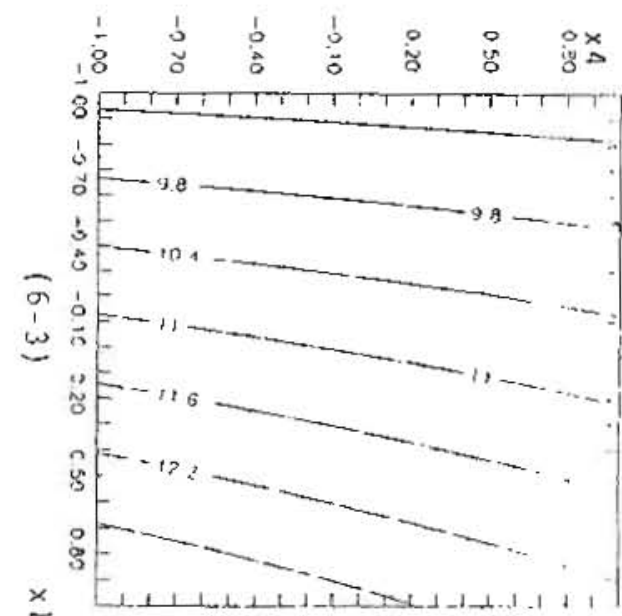




\subsection{Yarn Irregularity}

It can be nouced from the variance analysis that the main effect of yarn coum cradle spacing on yan irregulartity is heighly significant (at $99 \%$ confidence levei). As shown in Fig (3-1) using wide cradle spacing mcreases yan! irregularity (C. V\%) than that obtained with the closer one. This, may be attributed to a wide cradle spacing that will help in uncreasing the unmber of floatuig fibres which results in a high ırregularity.

The curves in Fig (6) show that, yarn regularity deteriorates with finer count than these with coarser count. The values of yam irregularity C.V\% ranged between $9.5 \%$ to $12 \%$ at lower spindle speed while it varies from $9 \%$ to $14 \%$ at higher spindle speed.

\subsection{Yarn Imperfections:}

From the statistieal analysis given in Tables $(5-a)$ and $(5-b)$, it can be seen that, yam count has a significant influence on yarn imperfections "neps, thick places and thin places at $99 \%$ "whatever the condition of other four paraneters. Also, the spindle speed affects significantly on neps and thin places at the levels of $99 \%$ and $90 \%$ respectively. In additon to the influence of the earlier parameters. yam imperfections were affected by apron spacing as shown in Fig. (3). Upper level of cradie spacing increased yann mperfections, this may be due to the incidcnce of slippage at wider apron spacing.

The two factor interactions, suci as, yan count with spindle speed, yan coun with top roller pressure and yari count with cradie spacing affect significntly neiss couml. as shown in Table $(5-a),(5-b)$ and $(6)$, as well as the interaction between spindle speed and cradle spacing. Also there are another two factor interactions, are given in table (5-1), (5-b) and (6), which have a significant effect on number of thin and thick places.

\section{4- Conclusion:}

The present study permits the following conclusions to be drawn:

l) It has been found that the parameters: yam linear density, spindle speed, traveller size, loading the top arm and cradle opening have a significant effect on yarn quality. Also, the interaction benween the effects of two paraneters is significant in the majorsty of the cases.

\section{ii) Yarn strength:}

Ring spun yam strength is infuenced by spmdle speed and cradle opening: The two factor interaction such as $X 1 \times 3, \times 2 \times 4$ and $X 1 \times 4$ affect significautly on yam strength..

A higher yam strength is oblained by close setting of cradle for fine and coarse yarns. 
For both fine and coarse yanis as loading the top ann mcreased yam strength decreased.

For both leveis of loading the top arm as the spmdle speed increased, the yarn strength decreased.

\section{(iii) Yarn elongation :}

The experiments clearly show that;

Higher spindle speed and yarn linear density affect significantly on yam eiongation.

Improper choice of traveller size for the linear density of required yam will reduce yan elongation.

(iv) Yarn uniformity:

A better rign spun yan uniformity was obtanned with cioser cradle opening.

For higher linear density a better unifonnity was achieved as the spundle speed increased. Whereas for lower linear density increasing spindle speed causes deterioration in yam evenness.

(v) Yarn Imperfections (thin, thick places and neps/1000m):

A constant relationsinip existed between yan imperfectuvis (thick, thun places and neps) and yarn linear density at varous spindle speed and cradle opening

Under the reported spmnning conditions it has been found that lower lincar densıty, higher spindle speed and as cradle opening is increased yarn irregularity and imperfections uncreased

\section{References:}

1-Sinpson $J$ and Sens C.L., Text ind., 123, 100 (Oct. 1959).

2- Simpson J., Callegan A. T. and Sens C.L., Text. ind. 124, 101,104,105 (Nov 1960)

3- Simpson J, Cailegan A. T. and Sens C.L., Text. Ind 124, 209 (May 1960)

4- Simpson J., Callegan A. T. and Sens C.L., Text. Ind. 125, 91 (June 1961)

5- Audivert R. and Vidella J. E., T. R. J 32, 652, 1962.

6. Rantnam T.V., Single Vs Double End Feed at the spinning frame, Procc. Fourti Tecinnol. Conf, 1962, Dec, 72.

7- Audivert R. and Vidella J. E., T. R. J. 33, 310, 1963.

8. Audivert R. and Vidella J. E., T. R. J. 33, 319,1963

9- Owenl- Davies, The Dseign and Analysis of Industral Experiments, 1963.

10. Shanklin E.H., Texl. World 114, 70 (Nov. 1964).

11. Yablonskii V., Tech. of Textrle Industry, U.S.S.R., No 4, 55, 1964.

12- Lous G. and Fion L. A, Textıle Bull., August, 1965.

13-Audivert R., Villaronga M. and Coscella R., T.R. 5. 37 , I, 1967.

14- Baiasubramaman N, T.R.J. 39, 155, 1969

15-El-Behery H.M., T.R.J. 41, 379, 1971.

16- Balasubramanian N., and Trivedi G. K, T.R. J. 43, 1. 1973.

17- Balasubramanan N., T. R. J. 45, 322, 1975

18- El-Bealy et al" Intenational Text. Eng Confg. ITEC " $89,23-25$, Dec. 1989, p. 135. 